\title{
Micro-Lasers on a Silicon Chip: Toward Silicon Photonics
}

\author{
Abstract: \\ Demonstration and performance of micro-lasers on a silicon micro-electronic chip are reviewed. \\ These devices use the Raman and parametric nonlinearities of thermal silica grown on silicon and \\ exhibit sub-100 microWatt thresholds at high quantum efficiency.
}

K. Vahala, T. J. Kippenberg, S. Spillane, D. Armani

Department of Applied Physics, California Institute of Technology

\section{(C)2005 Optical Society of America OCIS codes:, (230.5750) Resonators}

Recent reports of laser oscillation on a silicon wafer by way of the Raman effect highlight the interest in sources that can be processed on a silicon wafer $[1,2]$. Here, we describe a path to both Raman and parametric sources on silicon that utilizes thermal silica grown on the silicon substrate. This silica is processed into ultra-high-Q microresonators featuring Q factors in excess of 100 million [3] and cavity footprints that are typically less than 40 microns (see figures 1 and 2). The combination of ultra-high $\mathrm{Q}$ factor with the inherent micro-scale optical volumes of these resonators endow these devices with unique nonlinear performance. The optical threshold for onset of Raman oscillation is approximately 100 micoWatts (see figure 3), while their geometry enables the devices to be adapted for operation in the parametric regime [4]. We will review progress and recent results concerning first, the fabrication of ultra-high-Q silica micro-resonators on silicon wafers, and then, the adaptation of these resonators for use as both Raman and parametric sources. Fiber-coupled power in the $\mathrm{mW}$ att range with broadly tunable emission will be reviewed (see figure 4). In addition, we will describe a high efficiency coupling method (see figure 1) that enables the devices to be directly coupled to optical fiber $[5,6]$. The ability to leverage process technology available on silicon such as MEMs will also be illustrated (see figure 2). The fabrication of highefficiency micro-lasers on silicon is thus not only a step towards the long-term goal of silicon photonics, but in the short term can potentially provide a micro-scale silicon-alternative to conventional laser sources used in applications outside of communications.

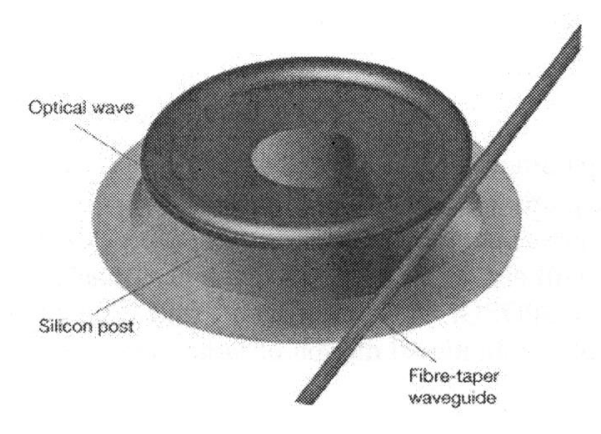

Figure 1: Rendering showing an ultra-high-Q toroidal microresonator coupling to a silica fiber taper waveguide.

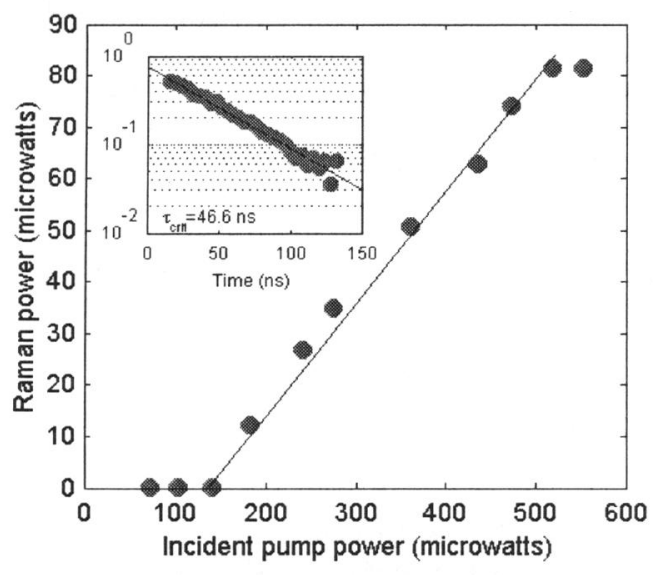

F Figure 3: Near threshold output power versus pump power plot for Raman laser illustrating threshold of 140 microWatts.

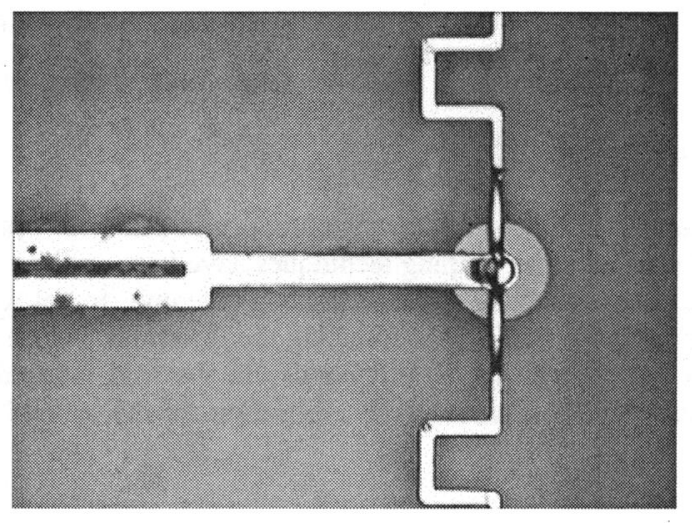

Figure 2: Microresonator attached to a MEMs control arm enabling micro-position control of the resonator relative to an optical waveguide.

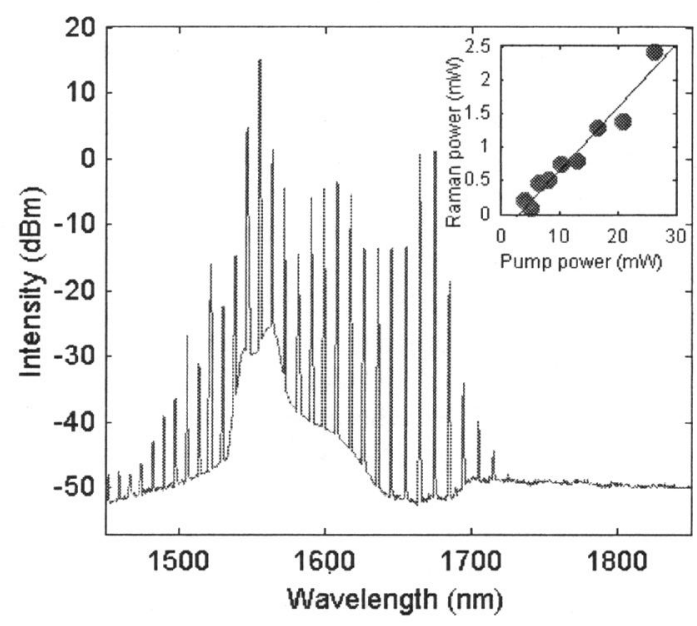

Figure 4: Spectrum showing broad-band Raman oscillation. Pump wave is at $1560 \mathrm{~nm}$ in the figure and several oscillation peaks are in excess of several mWatts (fiber coupled power). Inset is power output curve for one such peak. 
1. Kippenberg, T. J., Spillane, S. M., Armani, D. K. \& Vahala, K. J. Ultra-low-threshold Raman laser on a microelectronic chip. Optics Letters, vol 29, no. 11, June (2004).

2. H. Rong, A. Liu, R. Jones,O. Cohen, R. Nicolaescu, A. Fang, N. Panicia, "An all silicon Raman laser," Nature, Jan 13, 2005.

3. Armani, D. K., Kippenberg, T. J., Spillane, S. M. \& Vahala, K. J. Ultra-high-Q toroid microcavity on a chip. Nature 421, 925-928 (2003).

4. Kippenberg, T. J., Spillane, S. M. \& Vahala, K. J. Kerr nonlinearity optical parametric oscillation in an ultra-high-Q toroid microcavity. Physical Review Letters, vol 93, no. 8, August (2004).

5. Cai, M., Painter, O. \& Vahala, K. J. Observation of critical coupling in a fiber taper to a silica- microsphere whispering-gallery mode system. Physical Review Letters 85, 74-77 (2000).

6. Spillane, S. M., Kippenberg, T. J., Painter, O. J. \& Vahala, K. J. Ideality in a fiber-taper-coupled microresonator system for application to cavity quantum electrodynamics. Physical Review Letters 91, art. no.-043902 (2003). 